\title{
Clique cycle transversals in graphs with few $P_{4}$ 's
}

\author{
Raquel S. F. Bravo ${ }^{1 \dagger}$ Sulamita Klein $^{2 \ddagger}$ Loana Tito Nogueira $^{1 \S}$ Fábio Protti $^{1 \uparrow}$ \\ ${ }^{1}$ Fluminense Federal University, Niterói, RJ, Brazil \\ ${ }^{2}$ Federal University of Rio de Janeiro, RJ, Brazil
}

received $26^{\text {th }}$ Sep. 2012, revised 27th June 2013, 20 th Aug. 2013, accepted $16^{\text {th }}$ Sep. 2013.

A graph is extended $P_{4}$-laden if each of its induced subgraphs with at most six vertices that contains more than two induced $P_{4}$ 's is $\left\{2 K_{2}, C_{4}\right\}$-free. A cycle transversal (or feedback vertex set) of a graph $G$ is a subset $T \subseteq V(G)$ such that $T \cap V(C) \neq \emptyset$ for every cycle $C$ of $G$; if, in addition, $T$ is a clique, then $T$ is a clique cycle transversal (cct). Finding a cct in a graph $G$ is equivalent to partitioning $V(G)$ into subsets $\mathcal{C}$ and $\mathcal{F}$ such that $\mathcal{C}$ induces a complete subgraph and $\mathcal{F}$ an acyclic subgraph. This work considers the problem of characterizing extended $P_{4}$-laden graphs admitting a cct. We characterize such graphs by means of a finite family of forbidden induced subgraphs, and present a linear-time algorithm to recognize them.

Keywords: Clique, Cycle Transversal, Extended $P_{4}$-laden graph, Feedback Vertex Set

\section{Introduction}

A cycle transversal or feedback vertex set of a graph $G$ is a subset $T \subseteq V(G)$ such that $T \cap V(C) \neq \emptyset$ for every cycle $C$ of $G$. In other words, the removal of a cycle transversal leaves a graph without cycles. The problem of finding a minimum cycle transversal in an arbitrary graph $G$ is a classical NP-hard problem $[6,9,12]$ which has been extensively studied in several fields related to algorithms and complexity. It has first appeared within the context of combinatorial circuit design, and has applications in deadlock prevention in operating systems, database systems, genome assembly, constraint satisfaction, and Bayesian inference in artificial intelligence. We refer to the survey by Festa, Pardalos, and Resende [4] for further details on the algorithmic study of cycle transversal problems in a variety of areas, including approximation algorithms, linear programming and polyhedral combinatorics. In [1], the authors extend the NP-hardness of finding minimum cycle transversals in general graphs to bipartite graphs with maximum degree four. Algorithmic issues involving $C_{k}$ transversals (for fixed $k$ ) in graphs with bounded degree, where $C_{k}$ denotes a chordless cycle with $k$ vertices, are discussed in [8].

If a cycle transversal $T$ is also a clique, we say that $T$ is a clique cycle transversal, or simply cct. A graph admits a cct if and only if it can be partitioned into a complete subgraph and a forest; by this reason such a graph is called a $(\mathcal{C}, \mathcal{F})$-graph. Clique cycle transversals are studied in the more general context of graph partitions, especially sparse-dense partitions [3]: if $T$ is a cct in a graph $G$ then $G[T]$ is the 'dense' part, and $G-T$ the 'sparse' part. In [1], the authors describe a polynomial-time algorithm for recognizing graphs with cct, and a linear-time algorithm for cographs with cct based on a characterization of such cographs in terms of forbidden induced subgraphs. Distance-hereditary graphs with cct have been investigated in [2].

Denote by $P_{4}$ a graph with four vertices $a, b, c, d$ and edges $a b, b c, c d$. This work considers a class of graphs with few $P_{4}$ 's, the extended $P_{4}$-laden graphs. We say that a graph is extended $P_{4}$-laden if each of its induced subgraphs with at most six vertices that contains more than two induced $P_{4}$ 's is $\left\{2 K_{2}, C_{4}\right\}$-free. This class strictly contains the class of $P_{4}$-sparse graphs e, consequently, cographs. Our main result is a characterization of extended $P_{4}$-laden graphs with cct in terms of a family of twelve forbidden induced subgraphs. We also describe a lineartime algorithm for recognizing this class of graphs as a consequence of the given characterization. These results extend the previous ones for the class of cographs.

\footnotetext{
${ }^{\dagger}$ Email: raquelbr@cos.ufrj.br. Supported by CAPES and FAPERJ.

¥Email: sula@cos. ufrj.br. Partially supported by CNPq and FAPERJ.

$\S$ Email: loana@ic.uff.br. Partially supported by CNPq and FAPERJ.

TEmail: fabio@ic.uff.br. Partially supported by FAPERJ.
} 


\section{Background}

For standard definitions and notation in graph theory, see [11].

Extended $P_{4}$-laden graphs were characterized by [7] in terms of a decomposition technique and special graphs, called quasi-spiders and pseudo-split graphs. Extended $P_{4}$-laden graphs can be represented by a tree decomposition; the resulting tree is called the primeval tree.

We say that a graph $G$ is p-connected if for every partition of $V(G)$ into nonempty disjoint sets $A$ and $B$ there exists a crossing $P_{4}$, that is, a $P_{4}$ containing vertices from both $A$ and $B$. The p-connected components, or simply p-components, of a graph are the maximal induced subgraphs which are p-connected. It is worth mentioning that a p-component has either one vertex or at least four vertices [7]. A $p$-connected component $H$ of a graph $G$ is said to be trivial if $|H|=1$; otherwise, $H$ is nontrivial.

A p-connected graph $G$ is said to be separable if there exists a partition of $V(G)$ into nonempty disjoint sets $V_{1}$ and $V_{2}$ such that each $P_{4}$ which contains vertices from both sets has its endpoints in $V_{2}$ and its midpoints in $V_{1}$. In this case, we say that $G$ has a separation $\left(V_{1}, V_{2}\right)$.

The following theorem gives us information on the structure of extended $P_{4}$-laden graphs.

Theorem 1 [10] Let $G=(V, E)$ be a graph. Then exactly one of the following statements holds:

1. $G$ is disconnected;

2. $\bar{G}$ is disconnected;

3. There exists a unique separable p-component $H$ with separation $\left(H_{1}, H_{2}\right)$ such that every vertex outside $H$ is adjacent to all vertices in $H_{1}$ and to no vertex in $H_{2}$;

4. $G$ is p-connected.

Let $G_{1}=\left(V_{1}, E_{1}\right)$ and $G_{2}=\left(V_{2}, E_{2}\right)$ be disjoint graphs. The union and the join of $G_{1}$ and $G_{2}$ are graphs resulting, respectively, from the following operations:

- $G_{1} \cup G_{2}=\left(V_{1} \cup V_{2}, E_{1} \cup E_{2}\right)$

- $G_{1}+G_{2}=\left(V_{1} \cup V_{2}, E_{1} \cup E_{2} \cup\left\{x y \mid x \in V_{1}, y \in V_{2}\right\}\right)$

Operations $\cup$ and + are related to the first two cases of Theorem 1 .

Let $G_{1}=\left(V_{1}, E_{1}\right)$ be a separable p-connected graph with separation $\left(V_{1}^{1}, V_{1}^{2}\right)$ and $G_{2}=\left(V_{2}, E_{2}\right)$ be an arbitrary graph disjoint from $G_{1}$. The third case of Theorem 1 is represented by the following operation:

- $G_{1} \mathcal{P} G_{2}=\left(V_{1} \cup V_{2}, E_{1} \cup E_{2} \cup\left\{x y \mid x \in V_{1}^{1}, y \in V_{2}\right\}\right)$

As described by Jamison et al. [10], every graph $G$ is either a p-connected graph or can be uniquely obtained from its p-components and its weak vertices (i.e, those vertices that are not contained in any nontrivial p-component of $G$ ) by a finite sequence of operations $\cup,+$, and $\mathcal{P}$. Moreover, Theorem 1 suggests a way to represent an arbitrary graph $G$ by the primeval tree $T_{G}$, which is unique up to isomorphism. Each internal node $q$ of $T_{G}$ receives a label $i \in\{\cup,+, \mathcal{P}\}$. Such a label indicates that the subgraph associated with the subtree rooted at $q$ is obtained by performing an $i$-operation on the children of $q$. The leaves of the tree are the p-components of $G$.

Figure 1 represents a graph $G$ and its primeval tree $T_{G}$.

We say that $G$ is a spider if $V(G)$ can be partitioned into three subsets $\mathcal{S}, \mathcal{K}$, and $\mathcal{R}$ such that $\mathcal{S}$ is an independent set, $\mathcal{K}$ is a clique, $|\mathcal{S}|=|\mathcal{K}| \geq 2$, and there exists a bijective function $f: \mathcal{S} \rightarrow \mathcal{K}$ such that either $N_{G}(v)=\{f(v)\}$, for every $v \in \mathcal{S}$ (thin spider), or $N_{G}(v)=\mathcal{K}-\{f(v)\}$, for every $v \in \mathcal{S}$ (thick spider); in addition, every vertex in $\mathcal{R}$ is adjacent to every vertex in $\mathcal{K}$ and non-adjacent to every vertex in $\mathcal{S}$. We say that $\mathcal{S}$ and $\mathcal{K}$ are, respectively, the legs and body of the spider, and $\mathcal{R}$ is the head of the spider. Figure 2 depicts a thick and a thin spider.

A graph $G=(V, E)$ is split if $V$ can be partitioned into a clique $\mathcal{K}$ and an independent set $\mathcal{S}$ (with no restrictions on the edges between $\mathcal{S}$ and $\mathcal{K})$, i.e., $G=(\mathcal{S} \cup \mathcal{K}, E)$. For simplicity, we write $G=(\mathcal{S}, \mathcal{K})$ when the edge set $E$ is irrelevant for our purposes. It is worth observing that such a partition is not necessarily unique. It has been proved [5] that a graph $G$ is split if and only it does not contain $C_{5}, C_{4}$, or $\overline{C_{4}}=2 K_{2}$ as an induced subgraph.

Given a split graph $G=(\mathcal{S}, \mathcal{K})$, we say that $G$ is original if every vertex of $\mathcal{S}$ has a non-neighbor in $\mathcal{K}$ and every vertex of $\mathcal{K}$ has a neighbor in $\mathcal{S}$.

A graph $G$ is pseudo-split if its vertex set has a partition $(\mathcal{S}, \mathcal{K}, \mathcal{R})$ such that $\mathcal{S}$ is an independent set, $\mathcal{K}$ is a clique, and every vertex of $\mathcal{R}$ is adjacent to every vertex of $\mathcal{K}$ and non-adjacent to every vertex of $\mathcal{S}$, and $\mathcal{S} \cup \mathcal{K}$ induces a split graph. As for the spider, $\mathcal{S}, \mathcal{K}$, and $\mathcal{R}$ can be seen, respectively, as the legs, body and head of $G$; if $\mathcal{R}$ is empty, then $G$ is said to be a headless pseudo-split graph.

Observe that the complement of a pseudo-split graph is also a pseudo-split graph. Moreover, every spider is a pseudo-split graph. A quasi-spider is either a spider or a graph obtained from a spider $\mathscr{S}=(\mathcal{S}, \mathcal{K}, \mathcal{R})$ by 

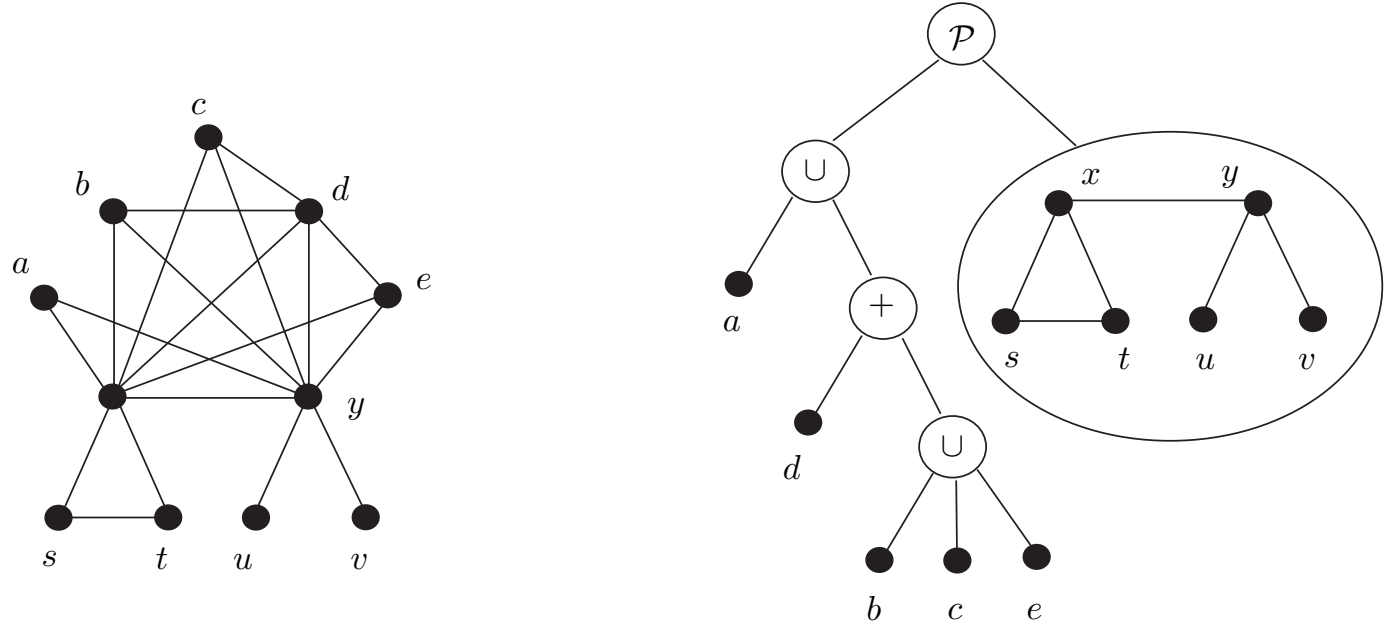

Fig. 1: A graph $G$ and its primeval tree $T_{G}$.

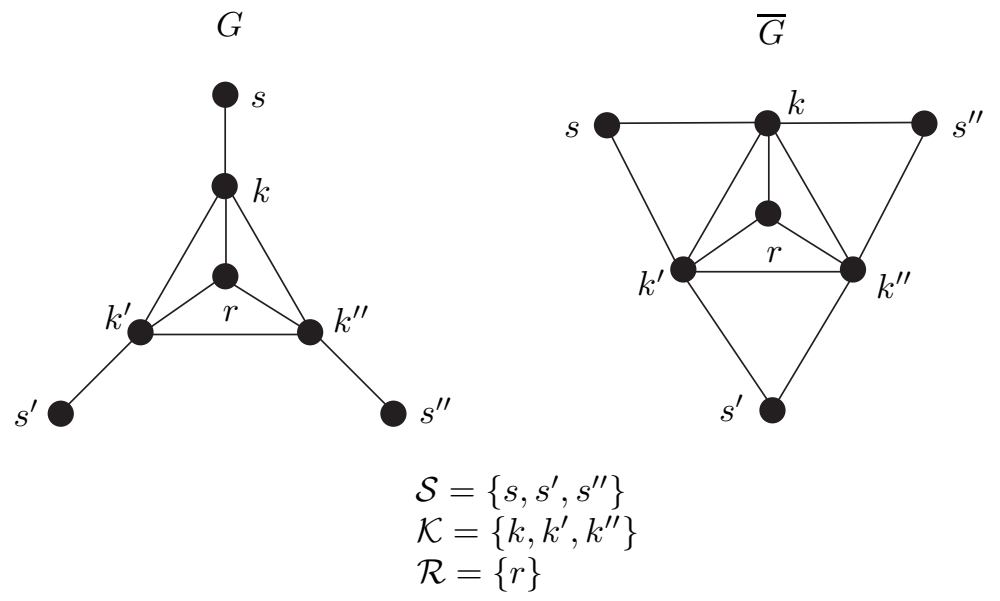

Fig. 2: $G$ is a thin spider and $\bar{G}$ is a thick spider.

replacing exactly one vertex $v \in \mathcal{S} \cup \mathcal{K}$ by a graph $H$ isomorphic to $K_{2}$ or $\overline{K_{2}}$, where vertices of $H$ have the same neighborhood as $v \in \mathcal{S} \cup \mathcal{K}$. The vertex $v$ that has been replaced by a $K_{2}$ or $I_{2}$ is called a q-vertex. A quasi-spider is a thin quasi-spider if it is originated from a thin spider, otherwise it is a thick quasi-spider. Figure 3 shows two examples of quasi-spiders: a thin one, with a vertex from set $\mathcal{S}$ replaced by $K_{2}$, and a thick one, with a vertex from set $\mathcal{K}$ replaced by $\overline{K_{2}}$.

The following theorem characterizes extended $P_{4}$-laden graphs.

Theorem 2 [7] $A$ graph $G$ is an extended $P_{4}$-laden graph if and only iffor every induced subgraph $H$ of $G$ exactly one of the following statements holds:

1. $H$ is disconnected;

2. $\bar{H}$ is disconnected;

3. $H$ is a quasi-spider whose head induces an extended $P_{4}$-laden graph;

4. $H$ is a pseudo-split graph whose head induces an extended $P_{4}$-laden graph;

5. $H$ is isomorphic to $C_{5}, P_{5}$ or $\overline{P_{5}}$;

6. $H$ is a trivial graph.

\section{Characterization}

Now we are ready to present the main result of this work.

Theorem 3 An extended $P_{4}$-laden graph $G$ admits a clique cycle transversal if and only if $G$ does not contain any of the graphs depicted in Figure 4 as an induced subgraph.

PROOF: By testing all possible cliques in each graph $H$ of Figure 4, we can see that none of them meets all the cycles in $H$. Below we apply this argument to each graph in Figure 4: 

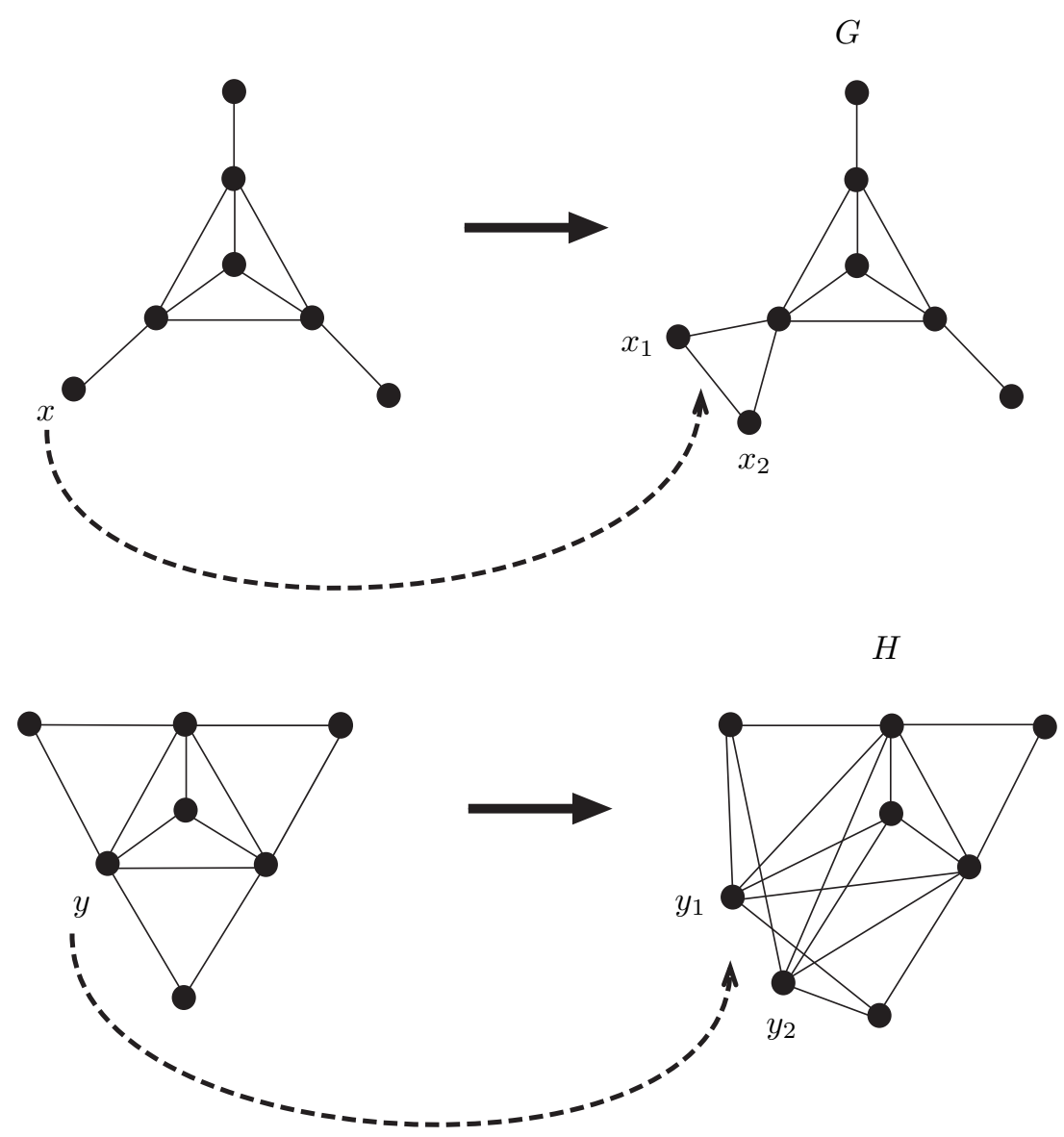

Fig. 3: $G$ is a thin quasi-spider and $H$ is a thick quasi-spider.

- $2 C_{3}$ : by removing any clique from this graph, there still remains a connected component which is a cycle; this argument also applies to the graphs $2 C_{4}, 2 C_{5}, C_{3} \cup C_{4}, C_{3} \cup C_{5}$, and $C_{4} \cup C_{5}$.

- $I_{2}+I_{2}+I_{2}$ : in this graph, any clique $C$ is formed by choosing at most one vertex of each independent set of size 2 ; thus, the vertices not chosen induce a graph that contains a $C_{3}$ as a subgraph.

- $2 K_{2}+I_{2}$ : any clique in this graph is formed by taking one or two vertices from a copy of $K_{2}$, plus at most one vertex lying outside that copy; thus, the remaining graph still contains a $C_{3}$.

- $I_{3}+I_{3}$ : since this graph is bipartite, any clique contains at most two vertices; by removing any vertex or edge, it is easy to see that there still remains an $I_{2}+I_{2}$ (i.e., a $C_{4}$ ) as a subgraph.

- $C_{5}+I_{2}$ : any clique in this graph is formed by choosing a vertex or an edge from the $C_{5}$, plus at most one vertex outside the $C_{5}$; after removing such a clique, the remaining graph contains a $C_{3}$ formed by an edge of the $C_{5}$ plus a vertex outside the $C_{5}$.

- $I_{3}+P_{4}$ : the argument for this graph is similar as above: cliques are formed by choosing a vertex or an edge from the $P_{4}$, plus at most one vertex outside the $P_{4}$; vertices not chosen induce a graph containing $I_{2}+K_{2}$ or $I_{2}+I_{2}$ as a subgraph.

- $P_{4}+P_{4}$ : in this graph, any clique $C$ is formed by taking a vertex or an edge of each $P_{4}$; thus, the removal of $C$ still a graph containing $K_{2}+K_{2}, K_{2}+I_{2}$, or $I_{2}+I_{2}$ as a subgraph, and all of them contain at least one cycle.

Hence, if $G$ contains some subgraph $H$ listed in Figure 4 as an induced subgraph then $G$ admits no cct. Conversely, suppose that $G$ contains no induced subgraph $H$ listed in Figure 4 as an induced subgraph, and suppose that $G$ admits no cct. In this case, $G$ contains a minimal induced subgraph $G^{\prime}$ with no cct, i.e., for every vertex $v \in V\left(G^{\prime}\right), G^{\prime}-v$ admits a cct. Without loss of generality, assume $G^{\prime}=G$. Consider the following three cases, according to Theorem 2: 

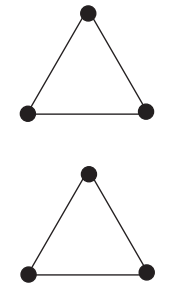

$2 C_{3}$

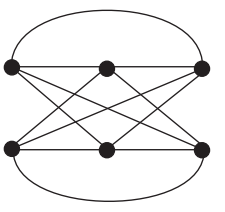

$I_{2}+I_{2}+I_{2}$
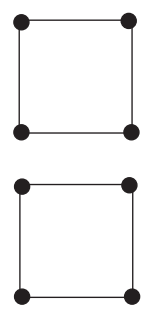

$2 C_{4}$

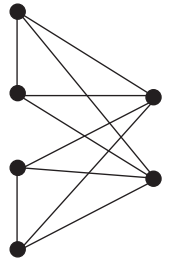

$2 K_{2}+I_{2}$
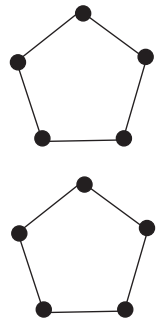

$2 C_{5}$

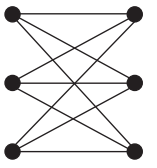

$I_{3}+I_{3}$
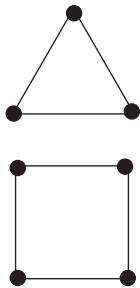

$C_{3} \cup C_{4}$
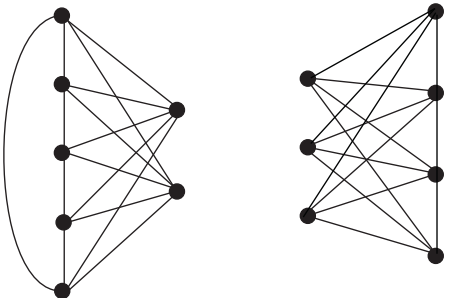

$I_{3}+P_{4}$
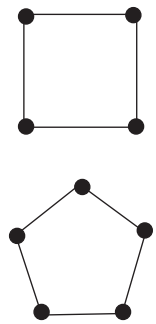

$C_{4} \cup C_{5}$

Fig. 4: Minimal forbidden subgraphs for extended $P_{4}$-laden graphs with cct.

CASE 1: $G$ is disconnected.

Write $G=G_{1} \cup G_{2} \cup \ldots G_{k}$, with $k \geq 2$ and each $G_{i}$ connected. By minimality of $G$, each $G_{i}$ admits a cct. Moreover, each $G_{i}$ is not a tree, otherwise $G^{\prime}=G-G_{i}$ would admit a cct, as well as $G=G^{\prime} \cup G_{i}$. Then, each $G_{i}$ must contain a cycle $\left(C_{3}, C_{4}\right.$, or $C_{5}$, since extended $P_{4}$-laden graphs contain no induced $C_{h}$ for all $\left.h \geq 6\right)$. It follows that $G$ contains one of the following graphs as an induced subgraph: $C_{3} \cup C_{3}, C_{3} \cup C_{4}, C_{3} \cup C_{5}, C_{4} \cup C_{4}$, $C_{4} \cup C_{5}$, or $C_{5} \cup C_{5}$. This is a contradiction.

CASE 2: $\bar{G}$ is disconnected.

Write $G=G_{1}+G_{2}+\cdots+G_{k}$, where $k \geq 2$ and $\overline{G_{i}}$ is connected for $1 \leq i \leq k$. If some $G_{i}$ is a complete subgraph, by minimality of $G$ the graph $G-G_{i}$ admits a cct, as well as $G$. Contradiction. Hence, assume that every $G_{i}$ contains a pair of non-adjacent vertices. If $k \geq 3$ then $G$ contains $I_{2}+I_{2}+I_{2}$ as an induced subgraph, a contradiction. If $k=2$, we consider three possibilities:

- CASE 2.1: Both $G_{1}$ and $G_{2}$ contain $I_{3}$. Then $G$ contains $I_{3}+I_{3}$ as an induced subgraph, a contradiction.

- CASE 2.2: Exactly one of $G_{1}, G_{2}$ contains $I_{3}$.

Without loss of generality, suppose that $G_{1}$ contains $I_{3}$. Subgraph $G_{2}$ cannot contain $C_{5}$, otherwise $G$ contains $I_{2}+C_{5}$ as an induced subgraph. In addition, $G_{2}$ cannot contain $P_{4}$, otherwise $G$ contains $I_{3}+P_{4}$ as an induced subgraph. Therefore, $\overline{G_{2}}$ does not contain $C_{3}, C_{5}$, and $P_{4}$, i.e., $\overline{G_{2}}$ is a triangle-free connected cograph, which implies that $\overline{G_{2}}$ is complete bipartite. We conclude that $G_{2}$ is a union of two disjoint, nonempty cliques $C$ and $C^{\prime}$. Assume that $|C| \geq\left|C^{\prime}\right|$. If $\left|C^{\prime}\right| \geq 2$ then $G$ contains $I_{2}+2 K_{2}$ as an induced subgraph. Thus $|C| \geq\left|C^{\prime}\right|=1$.

If $G_{1}$ is a split graph then $V\left(G_{1}\right)$ can be partitioned into an independent set $S_{1}$ and a clique $C_{1}$; hence $G$ admits cct $C \cup C_{1}$, a contradiction. Therefore, $G$ contains $I_{2}+C_{5}$ (if $G_{1}$ contains $C_{5}$ ), or $I_{2}+2 K_{2}$ (if $G_{1}$ contains $2 K_{2}$ ), or $I_{2}+I_{2}+I_{2}$ (if $G_{1}$ contains $C_{4}$ ) as an induced subgraph; hence, all these cases are contradictory.

- CASE 2.3: Both $G_{1}$ and $G_{2}$ do not contain $I_{3}$.

If $G_{1}$ or $G_{2}$ contains $C_{5}$ then $G$ contains $I_{2}+C_{5}$ as an induced subgraph. Therefore both $G_{1}$ and $G_{2}$ do not contain $C_{5}$. If both $G_{1}$ and $G_{2}$ contain $P_{4}$ then $G$ contains $P_{4}+P_{4}$ as an induced subgraph, a contradiction. Without loss of generality, suppose that $G_{2}$ does not contain $P_{4}$. Since $C_{5}$ and $P_{4}$ are self-complementary graphs, note that $\overline{G_{2}}$ does not contain $C_{3}, C_{5}$, and $P_{4}$; hence, $\overline{G_{2}}$ is a triangle-free connected cograph, as in Case 2.2. From this fact, we derive exactly the same contradictions as in that case.

CASE 3: $G$ and $\bar{G}$ are connected. By Theorem 2, the analysis of this case is as follows. 
If $G$ is trivial or $G=P_{5}$ or $G=\overline{P_{5}}$ or $G=C_{5}$ then $G$ admits a cct, which is a contradiction.

If $G$ is a pseudo-split graph whose vertex set has a partition $(\mathcal{S}, \mathcal{K}, \mathcal{R})$, where $\mathcal{R}$ is the head and induces an extended $P_{4}$-laden graph, then, by minimality of $G, G[\mathcal{R}]$ admits a cct, namely $C_{1}$. In this case, $G$ also admits a cct, namely $\mathcal{K} \cup C_{1}$, which is again a contradiction.

If $G$ is a quasi-spider whose head $\mathcal{R}$ induces an extended $P_{4}$-laden graph, we analyze three subcases:

- CASE 3.1: $G$ is a quasi-spider with a q-vertex $s \in \mathcal{S}$ replaced by either an $I_{2}$ or a $K_{2}$ with vertices $u, v$. In this case, the subset $(\mathcal{S}-\{s\}) \cup\{u, v\} \cup \mathcal{K}$ induces a subgraph with cct $\mathcal{K}$. Notice that, by minimality, $G[\mathcal{R}]$ admits a cct, say $C_{1}$. Consequently, $G$ admits a cct $\mathcal{K} \cup C_{1}$, since $(\mathcal{S}-\{s\}) \cup\{u, v\} \cup\left(\mathcal{R}-C_{1}\right)$ induces a forest. Contradiction.

- CASE 3.2: $G$ is a quasi-spider with a q-vertex $k \in \mathcal{K}$ replaced by a $K_{2}$ with vertices $u, v$. In this case, the subset $\mathcal{S} \cup(\mathcal{K}-\{k\} \cup\{u, v\}$ induces a subgraph with cct $(\mathcal{K}-\{k\}) \cup\{u, v\}$. By minimality, $G[\mathcal{R}]$ admits a cct $C_{1}$. Consequently, $G$ admits cct $(\mathcal{K}-\{k\}) \cup\{u, v\} \cup \mathcal{C}_{1}$, which is a contradiction.

- CASE 3.3: $G$ is a quasi-spider with a q-vertex $k \in \mathcal{K}$ replaced by an $I_{2}$ with vertices $u, v$. We analyze two additional possibilities:

- $G[\mathcal{R}]=(C, I)$ is a split graph, where $I$ is an independent set and $C$ is a clique. Then, $G$ admits a cct $(\mathcal{K}-\{u\}) \cup C$, because $\mathcal{S} \cup I \cup\{u\}$ induces a forest. This is a contradiction.

- $G[\mathcal{R}]$ is not a split graph, i.e., contains $2 K_{2}, C_{4}$, or $C_{5}$ as an induced subgraph. Then $G$ contains one of the following graphs as an induced subgraph: $I_{2}+2 K_{2}, I_{2}+I_{2}+I_{2}$, or $I_{2}+C_{5}$. Contradiction. This concludes the proof of the theorem.

\section{Recognizing extended $P_{4}$-laden graphs with cct in linear time}

The proof of the previous theorem directly gives a linear-time recognition algorithm for extended $P_{4}$-laden graphs with cct (Algorithm 1, described below).

The linear-time complexity is justified by the following facts:

(i) the primeval tree can be computed in linear time [10];

(ii) all the if-conditions in the algorithm can be checked in linear time.

In order to prove (ii), it suffices to show that the if-conditions based on recursive calls (lines 7, 13, 38, and 42) can be checked in linear time. (The remaining if-conditions are all easily checkable in linear time.) We use an inductive argument. First, it is clear that for a trivial graph one can check in linear time whether it admits a cct. Now we consider how recursive calls are made as Algorithm 1 traverses the primeval tree of the input graph $G$. In line 7 of the algorithm, a recursive call for each subtree associated with child subgraph $G_{i}$ of $G$ is made. In line 13 , a recursive call is made only for the subtree associated with child subgraph $G_{1}$. Finally, in lines 38 and 42 , a recursive call is made only for the subtree associated with child subgraph $G[\mathcal{R}]$. In any case, the recursive calls are made on subtrees corresponding to vertex-disjoint subgraphs of $G$. Assuming by induction that for graphs $H$ with fewer than $n$ vertices (where $n=|V(G)|$ ) one can check whether $H$ admits a cct in time linear in the size of $H$, we conclude that all the if-conditions in lines 7, 13, 38, and 42 can be checked in time linear in the size of $G$.

\section{Conclusions}

In this work, we use the structure of the extended $P_{4}$-laden graphs and its primeval tree decomposition to provide a characterization by forbidden subgraphs of extended $P_{4}$-laden graphs which admit clique cycle transversal, or, alternatively, which can be partitioned into a complete graph and a forest (acyclic graph). As a consequence, we develop a linear time recognition algorithm for this class of graphs. 


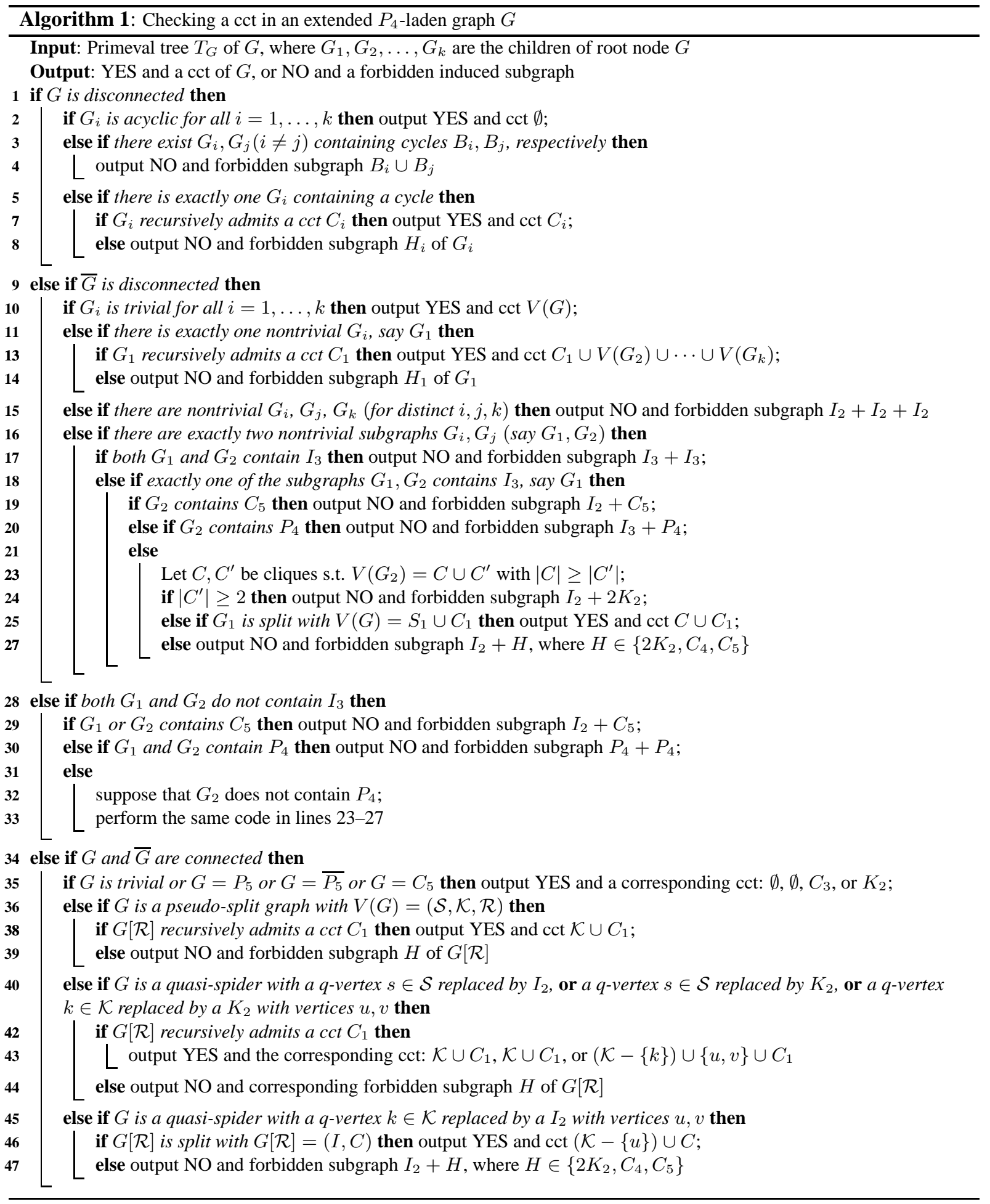




\section{References}

[1] Bransdstädt, A., Brito, S. P., Klein, S., Nogueira, L. T., and Protti, F. Cycle transversals in perfect graphs and cographs. Theoretical Computer Science 469 (2013) 15-23. See also Corrigendum, vol. 487 (2013) 103-105.

[2] Bransdstädt, A., Esposito, S., Nogueira, L. T., and Protti, F. Clique cycle transversals in distance hereditary graphs. VII Latin-American Algorithms, Graphs, and Optimization Symposium, Playa del Carmen, Mexico, april 2013. Electronic Notes in Discrete Mathematics, to appear.

[3] Feder, T., Hell, P., Klein, S., and Motwani, R. Complexity of graph partition problems. 31st Annual ACM Symposium on Theory of Computing, 464-472. Plenum Press, New York, 1999.

[4] Festa, P. and Pardalos, P. M., Resende, M. G. C. Feedback Set Problems. Encyclopedia of Optimization (2009), 1005-1016.

[5] Foldes, S., and Hammer, P. L. Split graphs. Proceedings of the Eighth Southeastern Conference on Combinatorics, Graph Theory and Computing (Louisiana State Univ., Baton Rouge, La., 1977), pp. 311-315. Congressus Numerantium, No. XIX, Utilitas Math., Winnipeg, Man., 1977.

[6] Garey, M. R., and Johnson, D. S. Computers and Intractability-A Guide to the Theory of NP-completeness, Freeman, San Francisco, 1979.

[7] Giakoumakis, V. $P_{4}$-laden graphs: a new class of brittle graphs. Information Processing Letters 60 (1996) $29-36$.

[8] Groshaus, M., Hell, P., Klein, S., Nogueira, L. T., and Protti, F. Cycle Transversals in Bounded Degree Graphs. Discrete Mathematics and Theoretical Computer Science 13:1 (2011) 45-66.

[9] Karp, R. M. Reducibility among combinatorial problems, Complexity of Computer Computations, R.E. Miller, J.W. Thatcher (eds.), Plenum Press (1972) 85-103

[10] Jamison, B., and Olariu, S. p-components and the homogeneous decomposition of graphs SIAM Journal Discrete Mathematics 8 (1995) 448-463.

[11] West, D. B. Introduction to Graph Theory. Prentice-Hall, New Jersey, 1996.

[12] Yannakakis, M. Node- and edge-deletion NP-complete problems. Proceedings of the Tenth Annual ACM Symposium on Theory of Computing - STOC'78, pp. 253-264, 1978, ACM Press. 